\title{
A useful framework for optimal replacement models
}

\author{
Terje Aven ${ }^{a}$ \& Rommert Dekker ${ }^{b}$ \\ " Stavanger University College, Ullandhaug, 4004 Stavanger, Norway \\ ${ }^{b}$ Erasmus University, Rotterdam, 3000 DR Rotterdam, The Netherlands
}

(Received 18 March 1996; revised 10 April 1997; accepted 15 May 1997)

\begin{abstract}
In this note we present a general framework for optimization of replacement times. It covers a number of models, including various age and block replacement models, and allows a uniform analysis for all these models. A relation to the marginal cost concept is described. (C) 1997 Elsevier Science Limited.
\end{abstract}

\section{INTRODUCTION}

Many papers have been written on maintenance optimization according to the various reviews, e.g., McCall [1], Pierskalla and Voelker [2], Sherif and Smith [3] and Valdez-Flores and Feldman [4]. Indecd, it is a fruitful area for mathematical research and the variety of systcms, deterioration and the way actions can be chosen allows a large number of models. Most of these models address the problem of optimizing the execution of a single action by which a full or part of a system is renewed and consider a single decision variable. Models with multiple components or multiple decision variables are much more difficult to analyze. Frequently the result of the single component models are used, see, e.g., Cho and Parlar [5].

There have been several attempts to structure the variety of models. Some of the overviews contain a qualitative description of the models, in terms of system, deterioration, knowledge and decision variables. Next there have been frameworks which contain several models. Aven and Bergman [6] published a quite general framework, which despite its generality does not seem very well known. As a consequence, the optimisation part of many papers in the field could have heen simplified and made more structured. Having future work in this area in mind, we think is it important to have at hand a set-up where the conditions and assumptions are formulated independent of particular models. Dekker [7] published a far more simpler variant, for which he was able to derive more results and make a link with multi-component systems. His approach is related to the marginal cost approach introduced by Berg [8,9].
A main idea behind these approaches was also presented in Zijlstra [10]. The advantages of these frameworks are the following: they indicate that ccrtain structure rcsults can be obtained, likc the characterization and structure of an optimal policy. They show that a certain type of analysis will be successful, though each time with other details. The same holds for the optimization method, see, e.g., Barros et al. [11]. The other advantage is that the framework can be used as building block for multi-component models, in which a variety of component types has to be addressed. The framework then allows the derivation of results for a class of models instead of just one. An interesting example is given in Dekker et al. [12], where the cyclic maintenance planning technique is applied to block type policies.

In this paper we extend the framework of Dekker [7] to incorporate more models, especially models where one has to wait for a suitable moment for preventive replacement. The framework is in fact a simpler version of the set-up presented in [6], which consider also condition based replacement policies. Since age is the only decision parameter in most replacement models in practice, it is useful to have at hand a framework restricting attention to such models.

We also give a marginal cost interpretation of the parameters of the framework. Compared to the traditional approach this allows for much faster derivation of results and a useful interpretation for practical application. Our results here are considered important because they provide a more formal link between the marginal cost function and the cost 
optimization function than what has been presented up to now in the literature.

The structure of the paper is as follows. In Section 2 we present the framework and its results. In the following sections we treat several models which are captured by the framework, like opportunity and minimal repair models.

\section{THE FRAMEWORK}

Consider a system and a decision variable $T$, which affects the timing of maintenance action(s) renewing the system. In the most simple case of the framework, $T$ represents the time at which the system is replaced preventively. Alternatively, $T$ may also be a critical time after which the first suitable moment (opportunity, failure) is awaited to renew the system. In both cases the 'no preventive maintenance' decision can then be represented by $T=\infty$. The problem is to determine the value of $T$ that optimizes a given objective function. We will consider both average and discounted costs. As the execution of the action(s) implies a renewal of the system we can apply renewal theory and obtain for the long-term average costs

$$
g(T)=\frac{C(T)}{H(T)},
$$

where $C(T)$ and $H(T)$ denote the expected cycle costs and length, respectively. We make the following assumptions.

\section{Assumptions}

1. Both $C(T)$ and $H(T)$ are absolute continuous functions of $T$, i.e. $C(T)=C(0)+\int_{0}^{T} c(t) \mathrm{d} t$ and $H(T)=H(0)+\int_{0}^{T} h(t) \mathrm{d} t$ for some functions $c(t)$ and $h(t)$.

2. $H(0) \geq 0, h(t) \geq 0$ for all $t>0$ and $c(t)=0$ if $h(t)=0$.

Absolute continuity is a stronger form of continuity and means that the function can be written as an indefinite integral of its derivative (which exists almost everywhere). If a function is differentiable everywhere and has a derivative which is integrable, then the function is absolutely continuous.

From the assumptions it follows that there exists a function $m(t)$ such that $c(t)=m(t) h(t), t>0$. To simplify notation we write $c$ for $C(0)$ and $d$ for $H(0)$. Accordingly, we can write $g(T)$ as

$$
g(T)=\frac{c+\int_{0}^{T} m(t) h(t) \mathrm{d} t}{\mathrm{~d}+\int_{0}^{T} h(t) \mathrm{d} t}, \quad T>0
$$

which form is put central in this paper. Notice that for $T=0$ the expected cycle cost is $c$, and typically this represents the cost of a preventive replacement. The quantity $m(t)$ can be interpreted as the expected deterioration cost rate and $h(t)$ often denotes a survival function, cf. the examples and the link to the marginal cost analysis given below. The framework covers quite some models. Below we list the most simple ones (see, e.g., Barlow and Proschan [13]), in the remaining sections we show that also more complex models can be incorporated

1. the block replacement model: in this case $c$ is the preventive replacement cost, $m(t)=k b(t)$ where $b(t)$ denotes the renewal density and $k$ the expected failure replacement costs. In the standard block replacement model, preventive replacements take no time $(d=0)$. Finally, $h(t)=1, t>0$, implying that a cycle always has length $T$;

2. the age replacement model: again $c$ denotes the preventive replacement cost, $h(t)$ is the survival function $1-F(t)$, where $F(t)$ is the cdf of the time to failure, $m(t)$ is the failure replacement costs $k$ times the hazard rate $z(t)=F^{\prime}(t) /(1-$ $F(t))$ and finally $d=0$;

3. the standard minimal repair model: again $c$ denotes the preventive replacement costs, $d=0$, $h(t)=1$ and $m(t)$ denote the expected costs due to minimal repairs, viz. $m(t)=c_{r} \lambda(t)$ where $\lambda(t)$ is the rate of occurrence of failures and $c_{r}$ is the cost of a minimal repair.

If we take $h(t)=1$ and $d=0$ we obtain the standard framework presented in Dekker [7]. In that case, the decision variable represents the interval at which the preventive replacement is carried out. The advantage of such a simpler framework is that preventive replacements can fully be planned and hence be harmonised in a multi-component case, see Dekker $t$ al. [12].

It turns out that for a large class of replacement models the optimality criterion total expected discounted cost can also be written in the form eqn (1). In this case, the numerator of eqn (1) represents the expected discounted cost associated with one replacement cycle and the denominator equals

$$
E\left(1-e^{-\alpha \tau(T)}\right)=\alpha E \int_{0}^{\tau(T)} e^{-\alpha t} \mathrm{~d} t
$$

where $\alpha$ is a positive discount factor and $\tau(T)$ the (stochastic) time to replacement, cf. e.g., Aven [14]. For example, in the simple block replacement model mentioned above we can write the expected discounted cost in the form (1) with $m(t)=$ $(k b(t) / \alpha)-c$ and $h(t)=\alpha e^{-\alpha t}$. Other examples are given in the following sections. 
The analysis of the framework follows similar lines as in Dekker [7] and Aven [14]. Notice that for every $T$ for which $C(T)$ and $H(T)$ have a derivative, we have

$$
\begin{aligned}
g^{\prime}(T) & =\frac{(m(T)-g(T)) h(T)}{H(T)} \\
& =\frac{h(T)}{H^{2}(T)}[\Psi(T)-c],
\end{aligned}
$$

where $\Psi(T)=m(T) H(T)-\int_{0}^{T} m(t) h(t) \mathrm{d} t$. Hence, $g^{\prime}(T)=0$ iff $m(T)-g(T)=0$ or $h(T)=0$. Alternatively, $g^{\prime}(T)=0$ iff $\Psi(T)=c$ or $h(T)=0$. It is seen that $\Psi(T)$ is increasing if $m(T)$ is. In Theorem 1 below we will now formulate the main results when $g(T)$ is decreasing, increasing and whether there exists an optimal policy. Notice that $h(T)=0$ implies that $g^{\prime}(T)=0$, but $g^{\prime}(T)$ does not necessarily need a change of sign. Accordingly we restrict ourselves to the case that $h(T)>0$. The proof of Theorem 1, as well as some remarks, are given in Appendix A.

Theorem 1. Suppose $h(T)>0$ for all $T>0$,

(i) if $m(T)$ is non-increasing on $\left[T_{0}, T_{1}\right]$ and $\Psi\left(T_{0}\right)<c$, then $g(T)$ is decreasing on $\left[T_{0}, T_{1}\right]$,

(ii) if $m(T)$ increases strictly for $T>T_{0}$, where $\Psi\left(T_{0}\right)<c$, and $\Psi\left(T_{1}\right)>c$ for some $T_{1}>T_{0}$, then $g(T)$ has a minimum, say $g^{*}$ in $T^{*}$, which is unique on $\left[T_{0}, \infty\right)$; moreover,

$$
m(T)-g(T) \begin{cases}<0 & \text { for } T_{0}<T<T^{*} \\ >0 & \text { for } T>T^{*}\end{cases}
$$

and

$$
m(T)-g^{*} \begin{cases}<0 & \text { for } T_{0}<T<T^{*} \\ >0 & \text { for } T>T^{*}\end{cases}
$$

If $g(t)$ is differentiable in $T^{*}$ then $m\left(T^{*}\right)=g^{*}$.

(iii) if $\Psi(T)<c$ for all $T>T_{0}$ then $g(T)$ is decreasing for $T>T_{0}$.

(iv) suppose $m(T)$ is increasing for $T>T_{0}$ and that $\Psi\left(T_{0}\right)<c$, then $\Psi\left(T_{1}\right)>c$ for some $T_{1}>T_{0}$ if one of the following conditions hold
(a) $\lim _{T \rightarrow x} m(T)=\infty$
(b) $\lim _{T \rightarrow \infty} m(T)>\lim _{T \rightarrow \infty} g(T)$
(c) $\lim _{T \rightarrow \infty} H(T)=\infty, \lim _{T \rightarrow \infty} m(T)=a$, for some $a>0$ and $\lim _{T \rightarrow \infty} \int_{0}^{T}[a-m(x)] h(x) \mathrm{d} x>c-a d$.

Theorem 1 implies that for optimization one only needs to consider those regions where $m(T)$ is increasing. Furthermore, it says that a myopic policy in which at every moment we consider whether to defer the replacement or not, is average optimal. That is, the expected cost of deferring the replacement to level $T+\Delta T$, being $m(T) h(T) \Delta(T)$, should be compared to the minimum average costs over an interval of the same length, being $g^{*} h(T) \Delta(T)$. Hence, if $m(T)$ is larger than $g^{*}$, the deferment costs are larger and we should replace. This result gives a structuring of the optimal policy and it gives an explanation of why a policy is optimal. Moreover, it allows us to define the so-called marginal cost analysis.

\subsection{Marginal cost analysis}

We will first present the analysis for the average cost criterion, the discounted cost criterion is explained later. An essential assumption behind the analysis is that the cycle length is an increasing function of the decision variable, i.e., if in a realization a cycle is stopped by decision variable $T_{1}$ it is stopped later by any decision variable $T_{2}>T_{1}$; this assumption holds for most replacement models. The analysis takes the following steps

1. identify the events which give rise to a system renewal

2. determine how the expected cycle length $H(T)$ and costs $C(T)$ change if the decision variable $T$ changes from $T$ to $T+\Delta T$, where $\Delta T$ is an infinitesimally small quantity. That is, consider a cycle end for decision variable $T$ and determine how the cycle would be extended if the decision variable would take the value $T+\Delta T$. Suppose that the expected lengthening of the cycle equals $h(T) \Delta T+o(\Delta T), \quad$ i.e., $\quad H(T+\Delta t)-H(T)=$ $h(T) \Delta T+o(\Delta T)$. Suppose next that the expected extra costs in case the cycle is extended amount to $c(T) \Delta T+o(\Delta T)$. Then we have $C(T+\Delta T)-C(T)=c(T) \Delta T+o(\Delta T)$, and it follows that $m(T)=c(T) / h(T)$.

In many cases we find $h(T)$ and $c(T)$ by conditioning on the event that the system has survived time $T$. Then $h(T)=\tilde{h}(T) S(T)$, where $\tilde{h}(T)$ equals the expected lengthening rate of the cycle given survival to $T$ and $S(T)$ represents the probability that the system survives $T$. Furthermore, $\quad c(T)=\tilde{c}(T) S(T)$, where $\tilde{c}(T)$ equals the expected cost rate given that the system has survived $T$. The function $m(T)$ is given by $m(T)=\tilde{c}(T) / \tilde{h}(T)$

3 . check the assumptions. If these hold, eqn (1) provides the average costs and Theorem 1 applies. In particular Theorem 1 states that the policy which replaces if $m(T)$ equals or is larger than $g(T)$ or $g^{*}$, is average optimal.

This approach was first introduced by Berg [8], although in a somewhat less formal way (he did not precisely state which conditions on $m(T)$ and $h(T)$ needed to be imposed and why it was correct). The advantage of the marginal cost approach over a traditional approach which first derives a formula for the average costs and then takes the derivative, is (i) it works much faster, because the formula for the average costs can be quite complex cf. Section 4 and Section 5 , and hence difficult to differentiate (ii) it 
provides an optimality equation with a very important interpretation for practical applications and it directly points at the crucial function $m(T)$. A large number of these models can be included in the framework presented in this paper. We shall demonstrate it in the following sections. But first we briefly show that it is possible to also give a marginal cost interpretation of the total discounted cost criterion.

A discounted cost rate $c(T)$ is found as in average cost case by extending the decision variable $T$ to $T+\Delta T$. As a simple illustration, consider the block replacement model mentioned above. Then clearly the discounted cost increases by $k b(T) \Delta T e^{-\alpha T}$ $c \alpha e^{-\alpha T} \Delta T+o(\Delta T)$ by deferring the replacement from $T$ to $T+\Delta T$. Hence the cost rate $c(T)$ cquals $(k b(T)-c \alpha) e^{-\alpha T}$.

Now consider the cycle length, which we denote $\tau(T)$. Introduce $\bar{H}(T)=E e^{-\alpha \tau(T)}$. We can interpret $\bar{H}(T)$ as the discounted value of the cycle length. Then we define the denominator rate $(h(T))$ in this case as $[\bar{H}(T+\Delta T)-\bar{H}(T)] / \Delta T$ as $\Delta T$ converges to zero, i.e. we consider an infinitesimally small increase in the decision variable $T$ and calculate the rate in discounted value of the cycle length. As an example, consider again the block replacement model. Clearly in this case the cycle length $\tau(T)$ equals $T$. Hence the change in the discounted value equals $\alpha \Delta T e^{-\alpha T}$, i.e., $h(T)=\alpha e^{-\alpha T}$, and thus $m(T)=(k b(T) / \alpha)-c$.

\section{MODEL OF BLOCK ET AL. [15]}

Block et al. introduced in [15] a model which includes several basic preventive maintenance models as special cases. The model is briefly described in the following.

Assume that a unit is minimally repaired or replaced according to the following scheme. The component is 'new' at time zero, and it is replaced whenever it reaches age $T$ (planned replacement). The case $T=\infty$ corresponds to no planned replacement. The expected cost of a planned replacement equals $u$. If the unit fails at age $t<T$, then it is either replaced by a new unit of the same type with probability $\alpha(t)$ (unplanned replacement) or it undergoes minimal repair with probability $\beta(t)=1-\alpha(t)$. Whether the component is replaced or minimally repaired at a failure may depend on a number of factors, such as the extent and cost of the repair, but 'on average' the unit is replaced at a failure at $t 100 \alpha(t) \%$ of the times and minimally repaired $100 \beta(t) \%$ of the times. The expected cost of an unplanned replacement is $v$. The expected cost of a minimal repair at time $t$ is $r(t)$. We assume negligible repair and replacement times.

Let $N(t)$ represent the number of minimal repairs in $[0, t]$, assuming that no replacement is performed in the time interval. The process $N(t)$ is assumed to be a non-homogeneous Poisson process with a failure intensity $\lambda(t)$.

The application of the framework now proceeds as follows, cf. step (II) of the procedure described in Section 2. Consider the unit having age $T$ and let us consider deferring the planned replacement to age $T+\Delta T$. First notice that to survive up to age $T$ there should be no failure and subsequent replacement up till age $T$, which occurs with probability $S(T)=$ $e^{-\int \delta \alpha(s) \lambda(s) d s}$ (a survival probability from a nonhomogeneous Poisson process). Given survival up to time $\mathrm{T}$ the cycle is extended to time $T+\Delta T$. Hence $\tilde{h}(T)=1$. We next determine the expected extra costs by extending the cycle given survival, i.e., $\tilde{c}(T)$, which consists of the costs duc to a failure occurring with probability $\lambda(T) \Delta T$. Given a failure the unit is minimally repaired with probability $\beta(T)$ against costs $r(T)$ and replaced with probability $\alpha(T)$ against costs $(v-u)$. Concluding, we have

$$
\begin{aligned}
h(T) & =S(T)=e^{-\int_{0}^{T} \alpha(s) \lambda(s) d s} \\
m(T) & =\tilde{c}(T)=[r(T) \beta(T)+(v-u) \alpha(T)] \lambda(T) \\
c & =u \\
d & =0 .
\end{aligned}
$$

We have thus by some rather straightforward arguments identified the key functions of the set-up of Section 2, and the conclusions of Section 2 follow, e.g.: If $m(T)$ is continuous, then $\Psi(T)$ is continuous and a finite, optimal $T^{*}$ satisfies $\Psi\left(T^{*}\right)-c=0$. If $m(T)$ is non-decreasing, then $g(T)$ is non-increasing for $T<T^{*}$ and non-decreasing for $T>T^{*}$. If $\Psi(T)$ is strictly increasing in $T$, then $T^{*}$ is unique and $g(T)$ is strictly decreasing for $T<T^{*}$ and strictly increasing for $T>T^{*}$.

Using the formal link established in Section 2 between the marginal cost functions and the average cost criterion, the analysis has been considerably simplified compared to the traditional analyses in $[14,15]$, which first derive a formula for the average cost criterion and then take the derivative.

Similar results can be shown for the total expected discounted cost. It is not difficult to see that in this case we obtain

$$
\begin{aligned}
h(T) & =\alpha e^{-\alpha T} S(T) \\
m(T) & =\frac{1}{\alpha}[r(T) \beta(T) \lambda(T)+(v-u) \alpha(T) \lambda(T)-u \alpha] \\
c & =u \\
d & =0 .
\end{aligned}
$$

\section{OPPORTUNITY-BASED AGE REPLACEMENT}

This model, which is referred to as the opportunity age replacement model (OARP), is presented in 
Dekker and Dijkstra [16]. We consider a unit with a stochastic lifetime $X$ with cumulative distribution function $F(t)$, probability density function $f(t)$ and failure rate function $z(t)$. The density function $f(t)$ is assumed to be continuous. Upon failure, the unit is replaced at cost $c_{f}$. A preventive replacement of the unit against cost $c_{p}$ is possible only at opportunities, which are supposed to be generated according to a Poisson process, independently of failures of the unit. Let the random variable $Y$ denote the time between successive opportunities, and let $E Y$ denote its finite mean. The problem is to determine a strategy for preventive maintenance which minimises the total long run (expected) cost per unit of time. Attention is restricted to the class of so-called age-based control limit policies. Under these policies a unit is preventively replaced at an opportunity if its age has passed the control limit $T$.

In [16] an expression for the optimality criterion is established and some optimality results shown. Using our framework the optirnisation could however been simplified and more structured.

According to step (II) of the procedure in Section 2, we consider again a change of the decision variable from $T$ to $T+\Delta T$. This will only effect the cycle length if an opportunity falls in the interval $[T, T+\Delta T]$, which occurs with probability $\Delta T / E Y$. In that case the cycle length is extended either to the first opportunity thereafter or to a failure, whichever occurs first. Let the r.v. $X_{t}$ denote the remaining lifetime beyond age $t$, i.e., $X_{t}=\max (X-t, 0)$. Hence the extension of the expected cycle length is $E\left(\min \left(X_{T}, Y\right)\right)$. The expected extra cost over that period equals $\left(c_{f}-c_{p}\right) P\left(X_{T}<Y\right) / E Y$ (only in case of a failure we have extra costs, otherwise we just do the preventive replacement). Accordingly we have

$$
\begin{aligned}
c & =c_{p}+\left(c_{f}-c_{p}\right) P(X<Y) \\
d & =E(\min (X, Y)) \\
h(T) & =E\left(\min \left(X_{T}, Y\right)\right) / E Y \\
c(T) & =\left(c_{f}-c_{p}\right) P\left(X_{T}<Y\right) / E Y .
\end{aligned}
$$

For a function $w(s)$ define

$$
V_{w}(T)=\int_{T}^{\infty} e^{-s \lambda} w(s) \mathrm{d} s
$$

where $\lambda=1 / E Y$. Then it follows that

$$
\begin{aligned}
h(T) & =\lambda e^{T \lambda} V_{(1-F)}(T) \\
m(T) & =\left(c_{f}-c_{p}\right) \frac{V_{f}(T)}{V_{(1-F)}(T)} \\
c & =c_{p}+\left(c_{f}-c_{p}\right) V_{f}(0) \\
d & =V_{(1-F)}(0) .
\end{aligned}
$$

The results of Section 2 now follow. It can be shown by differentiation that $m(T)$ is increasing (strictly increasing) if the hazard rate $z(T)$ has this property. We also see that $m(\infty)=z(\infty)\left(c_{f}-c_{p}\right)$. Hence since $g(\infty)=c_{f} / E X$, it follows that the condition $m(\infty)>$ $g(\infty)$ is equivalent to

$$
z(\infty)>c_{f} /\left(c_{f}-c_{p}\right) E X,
$$

which is seen to been identical to the condition stated in Theorem 3 of [16].

Alternatively, we could have derived these results by first establishing the average cost criterion. Let $Y_{T}$ denote the time from $T$ to the subsequent opportunity. Observing that

$$
g(T)=\frac{c_{p}+\left(c_{f}-c_{p}\right) \int_{0}^{\infty} P\left(t<T+Y_{T}\right) f(t) \mathrm{d} t}{\int_{0}^{\infty} P\left(t<T+Y_{T}\right)(1-F(t)) \mathrm{d} t}
$$

and $P\left(t<T+Y_{T}\right)$ equals $e^{-(t-1) \lambda}$ for $t \geq T$, and 1 for $t<T$, the desired expressions follow by straightforward differentiations of the numerator and the denominator with respect to $T$, and then writing these functions as the integrals of their derivatives.

It is left to the reader to establish similar results for the discounted case.

\section{OPPORTUNITY BASED BLOCK REPLACEMENT}

The opportunity block replacement model [17] is an extension of the block replacement model in which preventive replacement of a component may only be carried out at opportunities, which occurrence can be described by a renewal process with the generic r.v. $Y$ denoting the time between successive opportunities. Let $V(t)$ denote its distribution function, and let $c$ denote the cost associated with a replacement at an opportunity, and $c_{f}$ the cost induced by a failure with successive replacement. The decision variable $T$ is a control limit after which the first opportunity is used for replacing the component. In this case the long-term average costs amount to

$$
g(T)=\frac{c+c_{f} \int_{0}^{\infty} B(T+z) \mathrm{d} P\left(Z_{T} \leq z\right)}{T+E Z_{T}}
$$

where $B(T)$ denotes the renewal function corresponding to the components failures and the r.v. $Z_{T}$ the forward recurrence time of the opportunity process. In [17] the authors take much trouble to differentiate $g(T)$ and derive an optimality equation with a marginal cost interpretation. Here we can derive that directly by remarking that if the control limit $T$ is changed to $T+\Delta T$, we no longer use the opportunity occurring between $[T, T+\Delta T]$ but take the next one 
for replacement. Hence the extension of the expected cycle length equals $P\left(Z_{T} \leq \Delta T\right) E Y$ and the expected increase in the expected cycle costs $P\left(Z_{T} \leq \Delta T\right) c_{f}$ $\int_{0}^{\infty}[B(T+y)-B(T)] d V(y)$. Accordingly we have $d=E Z_{0}, h(T)=z_{Z_{T}} E Y$ and $m(T)=c_{f} / E Y \int_{0}^{\infty}[B(T+$ $y)-B(T)] d V(y)$, where $z_{Z_{T}}$ denotes the hazard or failure rate corresponding to $Z_{T}$. The optimality equation $m(T)=g^{*}$ can now be interpreted as the expected cost rate of deferring execution of preventive maintenance to the next opportunity equals the minimum average cost.

\section{MINIMAL REPAIR MODEL}

Consider a system which is subject to failures appearing according to a nonhomogeneous Poisson process with rate $\lambda(t)>0$. Upon failure the system can either be minimally repaired against costs $c_{r}>0$ or be replaced preventively against costs $c>0$. The problem is now to decide when to replace the system preventively. This model was first studied by Muth [18]. The optimal policy is of the form: 'replace the system preventively at the first failure after time $T$ '. The average costs $g(T)$ are given by the following formula:

$$
g(T)=\frac{c+c_{r} \int_{0}^{T} \lambda(t) \mathrm{d} t}{T+E W_{T}},
$$

where $E W_{T}$ denotes the expected time to the first failure after time $T$, in formula

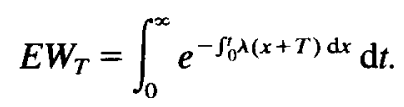

We will again demonstrate the usefulness of the marginal cost approach. Notice that by increasing $T$ to $T+\Delta T$ we only change the expected cycle length

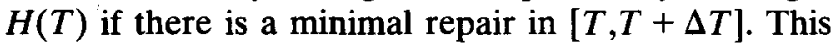
occurs with probability $\lambda(T) \Delta T$. In that case the cycle is extended with a time to the next failure, which expectation equals $E W_{T}$. Hence it easily follows that $h(T)=\lambda(T) E W_{T}$. In a similar way we obtain that $c(T)=c_{r} \lambda(T)$. Hence $m(T)=c_{r} / E W_{T}$. Finally we remark that $d=E W_{0}$. Note that $E W_{T}$ is decreasing if $\lambda(T)$ is increasing. We have shown that we have a special case of the set-up of Section 2.

\section{ACKNOWLEDGEMENT}

The authors would like to thank an anonymous referee for valuable comments and suggestions which improved the presentation of the paper.

\section{REFERENCES}

1. McCall, J. J., Maintenance policies for stochastically failing equipment: a survey. Management Science, 1965, 11, 493-524.
2. Pierskalla, W. P. and Voelker, J. A., A survey of maintenance models: the control and surveillance of deteriorating systems. Naval Research Logistics Quarterly, 1979, 23, 353-388.

3. Sherif, Y. S. and Smith, M. L., Optimal maintenance models for systems subject to failure-a review. Naval Research Logistics Quarterly, 1981, 28, 47-74.

4. Valdez-Flores, C. and Feldman, R. M., A survey of preventive maintenance models for stochastically deteriorating single-unit systems. Naval Research Logistics Quarterly, 1989, 36, 419-446.

5. Cho, D. I. and Parlar, M., A survey of maintenance models for multi-unit systems. European Journal of Operational Research, 1991, 51, 1-23.

6. Aven, T. and Bergman, B., Optimal replacement times-a general set-up. Journal of Applied Probability, $1986,23,432-442$.

7. Dekker, R., A general framework for optimisation, priority setting, planning and combining of maintenance activities. European Journal of Operational Research, $1995,82,225-240$.

8. Berg, M., A marginal cost analysis for preventive replacement policies. European Journal of Operational Research, 1980, 4, 136-142.

9. Berg, M., The marginal cost analysis and its application to repair and replacement policies. European Journal of Operational Research, 1995, 82, 214-224.

10. Zijlstra, M., Renewal replacement policies for one unit systems. European Journal of Operational Research, 1981, 8, 289-293.

11. Barros, A. I., Dekker, R., Frenk, J. B. G. and Van Weeren, S., Optimizing a general optimal replacement model by fractional programming techniques. Report, Econometric Institute, 9547/A, 1995, to appear in the Journal of Global Optimization.

12. Dekker, R., Van Egmond, R., Frenk, J. B. G. and Wildeman, R. M., A cyclic planning method for maintenance. Report, Econometric Institute, no. 9539/A, 1995.

13. Barlow, R. E. and Pruschan, F., Statistical Theory of Reliability and Life Testing. Holt, Rinehart \& Winston, 1975.

14. Aven, T., Reliability and Risk Analysis. Elsevier Publishing Company, 1992.

15. Block, H. W., Borges, W. S. and Savits, T. H., A general age replacement model with minimal repair. Naval Research Logistics, 1988, 35, 365-372.

16. Dekker, R. and Dijkstra, M. C., Opportunity-based age replacement: exponentially distributed times between opportunities. Naval Research Logistics, 1992, 39, 175-190.

17. Dekker, R. and Smeitink, E., Opportunity-based block replacement: the single component case. European Journal of Operational Research, 1991, 51, 46-63.

18. Muth, E. J., An optimal decision rule for repair vs. replacement. IEEE Transactions on Reliability, 1977, 26, 179-181.

\section{APPENDIX A}

\section{Proof of Theorem 1}

(i) If $m(T)$ is non-increasing, then $\Psi(T)$ is non-increasing and the result is immediate.

(ii) Since $\Psi\left(T_{0}\right)<c, \Psi(T)$ passes the level $c$ only 
once for $T>T_{0}$, which guarantees the uniqueness of the minimum. Equations (3) and (4) now follow immediately from eqn (2).

(iii) Follows directly from eqn (2).

(iv) Notice that $\Psi(T)-\Psi\left(T_{0}\right)=\int_{T_{0}}^{T}(m(T)-$ $m(x)) h(x) d x+\left[m(T)-m\left(T_{0}\right)\right] H\left(T_{0}\right)$. Hence, $\Psi(T)$ goes to infinity if $m(T)$ does so (case (a)). If condition b) holds it follows that for large $T$ we have $m(T)-g(T)>0$, which is equivalent to $\Psi(T)>c$. To establish $\mathrm{c}$ ), observe that $g(T)$ approaches $a$ from below if $c-a d+\int_{0}^{\infty}[a-m(T)] h(T) d T<0$.

\section{Remarks}

1. It will be clear that if $m(T)$ is constant on an interval, there may be multiple minima on that interval.

2. If condition b) of iv) holds, then we can conclude that there exists finite $T^{*}$ minimizing $g$ without assuming that $m(T)$ is increasing. Assuming $c>0$ and $d=0$, the condition $\Psi\left(T_{0}\right)<c$ trivially holds for small values of $T_{0}$ since $\Psi(0)=0$.

3. The case that $h(T)=0, T>T_{2}$ for some $T_{2}>0$, can be treated in a similar way, the condition in case (ii) that $\Psi\left(T_{1}\right)>c$ for some $T_{1}$ then changes into the condition that $\Psi\left(T_{2}\right)>c$.

4. The conditions (a) - (c) in (iv) have been added to show the correspondence with existing conditions for optimality in standard models. For example in the age replacement model we have case (b). The condition comes down to $\lim _{T \rightarrow \infty}\left(c_{f}-c_{p}\right) z(T)>c_{f} / E X$ where $E X$ denotes the expected lifetime and $z(T)$ denotes the hazard or failure rate of the item to be replaced. This condition is also given in [13]. In case of the block replacement model we have case (c). Here $H(T)=T$ and from renewal theory we obtain that $a=c_{f} / E X$. Now from the asymptotic expression of the renewal function we find that $\lim _{T \rightarrow \infty} \int_{0}^{T}[a-m(t)] h(t) d t=c_{f} 1 / 2\left(1-c_{X}^{2}\right)$

where $c_{X}^{2}$ represents the coefficient of variation. Accordingly the condition comes down to $1 / 2 c_{f}\left[1-c_{X}^{2}\right]>c_{p}$, which is exactly the condition in [13].

5. The results in (i) and (ii) can form the basis of an efficient optimization procedure. If it is easy to calculate $m(T)$ then a simple bisection procedure is quite efficient in solving $m(T)=g(T)$; for more details see Barros et al. [11]. 